\title{
Changes in left ventricular free wall thickness in patients with ischaemic heart disease
}

\author{
D. G. GIBSON, T. A. TRAILL, AND D. J. BROWN \\ From the Cardiac Department, Brompton Hospital, London
}

Continuous estimates of left ventricular free wall thickness were made from angiograms in 10 normal subjects and 20 patients with ischaemic heart disease. The time relations and extent of changes in wall thickness were compared with those in cavity area and transverse dimension, during isovolumic relaxation. In normal subjects, there was a mean reduction in wall thickness at this time of $0 \cdot 3 \pm 0.1 \mathrm{~cm}$ (mean \pm 1 standard deviation), but maximum rate of wall thinning occurred after mitral valve opening, during the phase of rapid ventricular filling. In 10 patients with ischaemic heart disease and regions of abnormal outward movement of endocardium during isovolumic relaxation, there was a reduction in wall thickness of $0 \cdot 8 \pm 0 \cdot 1 \mathrm{~cm}$ before mitral valve opening $(P<0.001$ with respect to normal), with little change during the remainder of diastole. In 4 patients with regions of inward movement of endocardium during isovolumic relaxation, there was an abnormal increase in wall thickness of $0.5 \pm 0.2 \mathrm{~cm}$ at this time $(P<0.001$ with respect to normal), though the wall behaved normally after mitral valve opening. In 6 patients, endocardial movement and wall thickness changes were normal. It is concluded that changes in left ventricular cavity shape occurring during isovolumic relaxation in patients with coronary artery disease result from abnormal wall thickness changes related to the presence of regional ischaemia.

Changes in left ventricular wall thickness during the cardiac cycle can be measured by angiography or echocardiography, and several studies have described findings in normal subjects, and abnormalities in patients with left ventricular hypertrophy or ischaemic heart disease (Rackley et al., 1964; Eber et al., 1969; Hugenholtz et al., 1969; Dodge et al., 1974; Dumesnil et al., 1974; Gould, et al., 1976). There is, however, little information on the relation between changes in wall thickness and those of the left ventricular cavity, either concerning their extent or their timing. Since the thickness of the myocardium is considerable, particularly at end-systole when it may approximate to that of the transverse diameter of the left ventricle in normal subjects, it is clear that abnormalities of wall dynamics might have significant effects on cavity size and shape. The present study was, therefore, designed to measure, from angiograms, the relative effects of wall thickness changes and movement of the epicardium in determining the position of the endocardium of the anterior wall of the left ventricle throughout the cardiac cycle. Using an extension of methods devised to study regional abnormalities of wall movement (Gibson et al., 1976), it was possible to investigate the Received for publication 10 March 1977 interrelation between wall thickness and cavity size and shape in normal subjects and patients with ischaemic heart disease.

\section{Subjects and methods}

\section{DEFINITION OF TERMS}

The left ventricular wall was defined as the structure lying between the epicardial and endocardial boundaries shown by the left ventriculogram. Wall thickness was defined as the distance between these boundaries, in a direction perpendicular to the endocardium at the site at which measurements were made, and the rate of change of wall thickness as the first derivative of this distance with respect to time. Regional changes in wall thickness measured in this way are referred to as wall dynamics (Dumesnil et al., 1974). These definitions are consistent with the procedure employed, which is one of image analysis rather than a direct study of anatomical structures.

SUBJECTS

Thirty patients were studied by left ventriculography and coronary arteriography for pain thought to result from ischaemic heart disease. Twenty of them proved to have obstructive coronary artery 
disease, and in the remainder, haemodynamics, left ventriculogram, and coronary arteriograms were all normal. The latter group, termed 'normal', served as the basis for comparison with patients with ischaemic heart disease.

Left ventriculograms were obtained either in the posteroanterior or $30^{\circ}$ right anterior oblique projection, using techniques that have previously been described (Gibson et al., 1976). Coronary arteriography was then performed using Judkins' technique, when multiple views of the right and left coronary arteries were obtained. Only left ventriculograms showing clear delineation of endocardium and epicardium along the free heart border were studied.

\section{ANALYSIS OF ANGIOGRAMS}

Left ventriculograms were analysed using an extension of computer methods previously described (Gibson et al., 1976), which aimed at delineating changes in left ventricular cavity and wall thickness, frame by frame. For each cine frame, the outline of the cavity was digitised, followed by a second line, representing the epicardial surface of the free wall over the region visible on the angiogram. This information, along with a fixed external reference point on the margin of the cine frame and calibration data from a grid exposed immediately after the angiogram at mid-chest level, was stored on disc for further calculations. Cavity area was calculated by numerical integration. In the group of patients with ischaemic heart disease, endocardial movement along the anterior wall was analysed using methods previously described. Of the 20 patients with ischaemic heart disease, there were: (1) 6 patients in whom anterior wall movement was normal with respect to both amplitude and timing; (2) 2 patients with only hypokinesis of the anterior wall; (3) 10 patients who had regions of abnormal outward movement before mitral valve opening: in all these patients, comparison could be made with a normally moving region of anterior wall on the same ventriculogram; and (4) 4 patients who had regions of abnormal inward movement before mitral valve opening: of these 1 also showed anterior hypokinesis and 2 abnormal outward movement elsewhere along the anterior wall.

Wall thickness changes were studied in two ways:

(1) The digitised outlines of cavity and epicardium for the beat to be studied were displayed on a Tektronix 4010 computer controlled display. A straight line was selected across the cavity so as to pass through the free wall and the cavity itself, approximately perpendicular to the walls. Plots were then made of changes with time of dimension and wall thickness and their corresponding rates of change. The time of mitral valve opening, determined as the frame in which unopacified dye from the left atrium first appeared within the left ventricular cavity, was superimposed on all traces, along with that of minimum cavity area. A representative example is shown in Fig. 1. From these plots, the following measurements were made: (a) enddiastolic and end-systolic wall thickness and cavity dimension, together with their peak rates of change during systole and diastole, (b) the extent of wall thinning between the time of maximum thickness and mitral valve opening (Table, 1), and

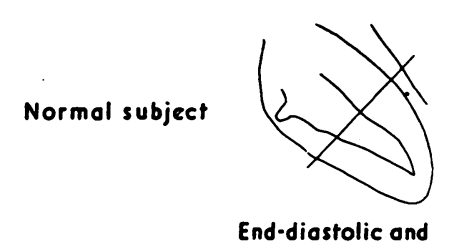

End-systolic fromes

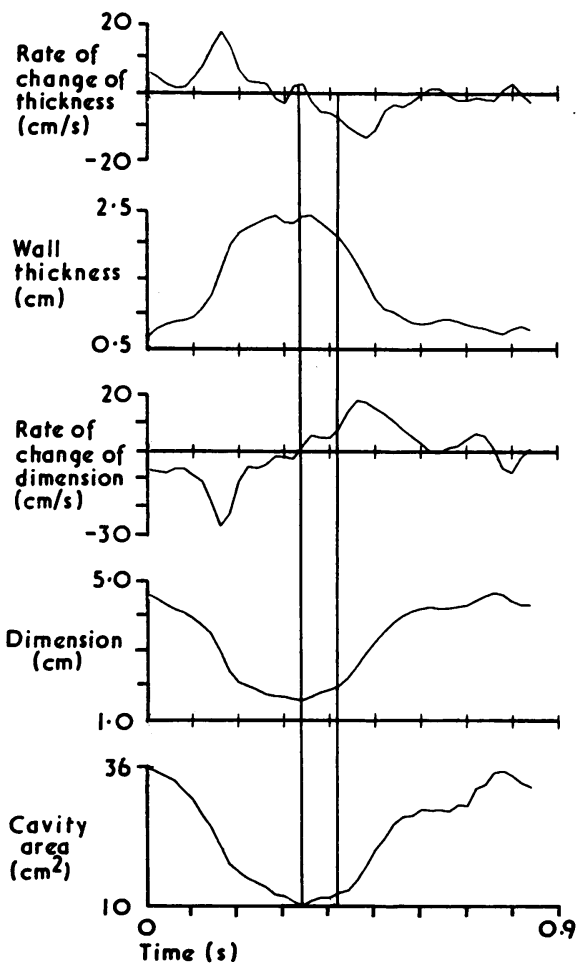

Fig. 1 Measurements of cavity area, transverse dimension, and wall thickness in a normal subject. The vertical lines mark the times of minimum cavity area and mitral valve opening. The position of the transverse dimension and of the free wall epicardium digitised are indicated in relation to end-diastolic and end-systolic cavity outlines. 
Table

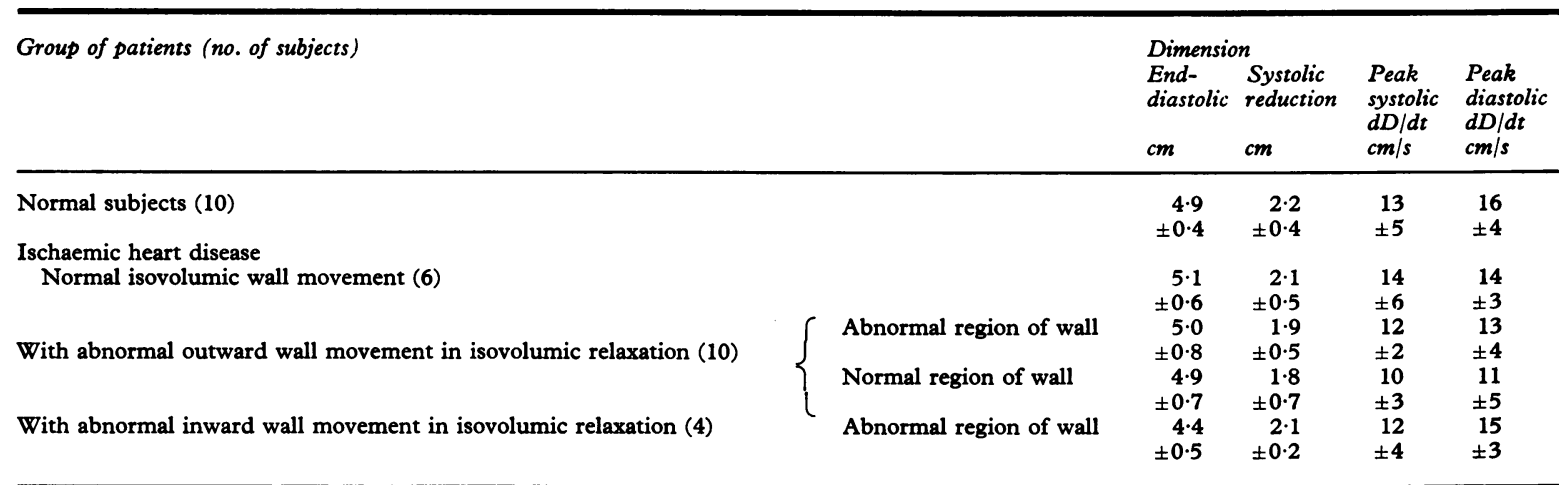

1, wall thinning before mitral valve opening ( $\mathrm{mm}) ; 2$, maximum wall thickness to minimum cavity area (ms); 3 , mitral valve opening to peak thinning rate (ms); 4, rapid thinning period (ms); 5, maximum wall thickness to peak thinning rate (ms).

*Significantly different from normal subjects $(P<0.01$ or 0.001$)$.

the duration of the rapid thinning period, defined as the time interval between maximum wall thickness and the discontinuity marking the end of rapid thinning visible on all traces (Table, 4). The time interval from maximum wall thickness to the time of peak wall thinning rate was also recorded (Table, 5); (c) the relation between changes in wall thickness and events referable to the cavity as a whole was studied in terms of the interval between maximum wall thickness and minimum cavity area (Table, 2), and between mitral valve opening and the time of peak wall thinning rate (Table, 3); and (d) the change in transverse dimension between end-systole and end-diastole was compared with the corresponding change in wall thickness.

(2) The second method of display was aimed at providing a more comprehensive picture of local abnormalities of wall dynamics. This was based on a method devised for studying local movement around the endocardium of the left ventricular cavity. Forty equally spaced points were defined around the end-diastolic endocardial outline, starting from the border of the aorta adjacent to the mitral valve and proceeding anticlockwise, and from each the nearest point on the end-systolic outline was sought. Along each of the lines thus defined, endocardial position was measured for each cine frame, relative to the end-diastolic position, resulting in 40 plots of endocardial position against time throughout the cardiac cycle. In the region of free wall for which the epicardium was also digitised, these same lines were continued outwards, and the distance along them between endocardial and epicardial outlines measured, and also plotted against time throughout the cardiac cycle. Information about endocardial position was displayed as an isometric plot and identical methods were used to display changes in wall thickness, examples being given in Fig. 2 and 4.

\section{Results}

The results are given in detail in the Table.

NORMAL SUBJECTS

In the normal patients, end-diastolic dimension, measured at the level of the mitral valve, was $4.9 \pm 0.4 \mathrm{~cm}$, with a reduction of $2.2 \pm 0.4 \mathrm{~cm}$ during systole. Peak systolic rate of shortening was $13 \pm 5 \mathrm{~cm} / \mathrm{s}$, and increase during diastole was $16 \pm 4 \mathrm{~cm} / \mathrm{s}$. End-diastolic wall thickness was $0.9 \pm 0.2 \mathrm{~cm}$ at this level of the ventricle, increasing to $2.0 \pm 0.5$ at end-systole. The peak rate of increase in wall thickness was $9 \pm 4 \mathrm{~cm} / \mathrm{s}$, and maximum wall thickness was synchronous ( $\pm 15 \mathrm{~ms}$ ) with minimum cavity area (Table, 2 and Fig. 1). The peak rate of wall thinning was $10 \pm 4 \mathrm{~cm} / \mathrm{s}$ and a distinct rapid thinning period could be identified, starting at the time of maximum wall thickness and ending with a discontinuity on the trace of the first derivative of wall thickness with time (Table, 4). This lasted $220 \pm 40 \mathrm{~ms}$, with maximum rate of wall thinning occurring $110 \pm 40 \mathrm{~ms}$ after maximum wall thickness (Table, 5). During the interval between minimum cavity area and mitral valve opening, wall thickness fell by $0 \cdot 3 \pm 0 \cdot 1 \mathrm{~cm}$ (Table, 1). Mitral valve opening preceded peak thinning rate in 8 out of 10 patients, by $30 \pm 30 \mathrm{~ms}$ for the group as a whole (Table, 3). In these patients, changes in wall thickness were uniform throughout the cardiac cycle over the region of wall that could be studied. A representative isometric plot is shown in Fig. 2. There is uniform thickening during systole, slight thinning with isovolumic 


\begin{tabular}{|c|c|c|c|c|c|c|c|c|}
\hline $\begin{array}{l}\text { Wall thic } \\
\text { End- } \\
\text { diastolic } \\
\mathrm{cm}\end{array}$ & $\begin{array}{l}\text { ckness } \\
\text { End- } \\
\text { systolic } \\
\text { cm }\end{array}$ & $\begin{array}{l}\text { Peak } \\
\text { systolic } \\
d W / d t \\
\mathrm{~cm} / \mathrm{s}\end{array}$ & $\begin{array}{l}\text { Peak } \\
\text { diastolic } \\
d W / d t \\
\mathrm{~cm} / \mathrm{s}\end{array}$ & $\begin{array}{l}1 \\
\mathrm{~mm}\end{array}$ & ${ }_{2}^{T i m e s}$ & $\begin{array}{c}\text { (ms) } \\
3\end{array}$ & 4 & 5 \\
\hline $\begin{array}{r}0.9 \\
\pm 0.2\end{array}$ & $\begin{array}{r}2.0 \\
\pm 0.5\end{array}$ & $\begin{array}{r}9 \\
\pm 4\end{array}$ & $\begin{array}{r}10 \\
\pm 4\end{array}$ & $\begin{array}{r}3 \\
\pm 1\end{array}$ & $\begin{array}{r}0 \\
\pm 15\end{array}$ & $\begin{array}{r}30 \\
\pm 30\end{array}$ & $\begin{array}{r}220 \\
\pm 40\end{array}$ & $\begin{array}{r}110 \\
\pm 40\end{array}$ \\
\hline $\begin{array}{r}0.8 \\
\pm 0.3 \\
0.8 \\
\pm 0.2 \\
0.9 \\
\pm 0.2 \\
0.8 \\
\pm 0.2\end{array}$ & $\begin{array}{r}1.8 \\
\pm 0.3 \\
1.8 \\
\pm 0.2 \\
2.1 \\
\pm 0.3 \\
2.0 \\
\pm 0.4\end{array}$ & $\begin{array}{r}10 \\
\pm 5 \\
7 \\
\pm 2 \\
8 \\
\pm 1 \\
7 \\
\pm 2\end{array}$ & $\begin{array}{r}8 \\
\pm 2 \\
11 \\
\pm 2 \\
9 \\
\pm 3 \\
9 \\
\pm 1\end{array}$ & $\begin{aligned} & 4 \\
& \pm 2 \\
& 8^{\star} \\
& \pm 1 \\
& 3 \\
& \pm 1 \\
&-5^{\star} \\
& \pm 2\end{aligned}$ & $\begin{array}{r}0 \\
\pm 10 \\
+10 \\
\pm 20 \\
0 \\
\pm 30 \\
-80^{\star} \\
\pm 20\end{array}$ & $\begin{array}{c}20 \\
\pm 40 \\
-80^{\star} \\
\pm 40 \\
40 \\
\pm 40 \\
10 \\
\pm 20\end{array}$ & $\begin{array}{c}210 \\
\pm 40 \\
120^{\star} \\
\pm 20 \\
200 \\
\pm 60 \\
190 \\
\pm 70\end{array}$ & $\begin{array}{c}110 \\
\pm 30 \\
60 \star \\
\pm 20 \\
110 \\
\pm 40 \\
80 \\
\pm 20\end{array}$ \\
\hline
\end{tabular}

Regional woll thickness

Normol subject

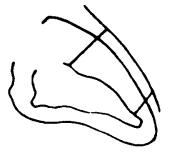

End-diastolic and

End-systolic fromes

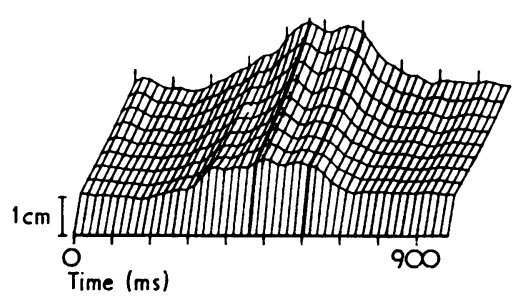

Fig. 2 Multiple plots of wall thickness against time from a normal subject, showing pattern of wall dynamics along the region of free wall from the apex (front) towards the aortic root (back), as in the upper panel which shows end-systolic and end-diastolic outlines.

Diagonal lines represent timing of successive cine frames, and accentuated lines minimum cavity area and mitral valve opening. Maximum wall thickness in individual plots is indicated by small vertical lines.

relaxation, and a greatly increased rate of thinning after mitral valve opening. These appearances were seen in all normal subjects.

\section{ISCHAEMIC HEART DISEASE}

In 6 patients, left ventricular wall movement was normal, and in these patients, changes in wall thickness were also within normal limits, the results being shown in Table, 1 .

Two patients showed hypokinesis of the anterior wall. End-diastolic wall thickness was normal in both, but the increases with systole were reduced to $0.3 \mathrm{~cm}$ and $0.5 \mathrm{~cm}$, as were peak values of thickening to 2 and $4 \mathrm{~cm} / \mathrm{s}$, respectively. Neither patient showed a period of rapid thinning or significant change in wall thickness during the period of isovolumic relaxation.

Ten patients had normal systolic amplitude of wall movement, but excessive outward movement during isovolumic relaxation, in part of the free wall (Fig. 3). In these patients, it was possible to compare wall thickness dynamics of a normal region with those from a region where they were abnormal. In regions defined as normal, with respect to movement of endocardium, wall thickness changes were not significantly different in any way from

Ischaemic heart disease

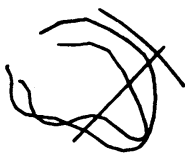

End-diastolic and End-systolic frames

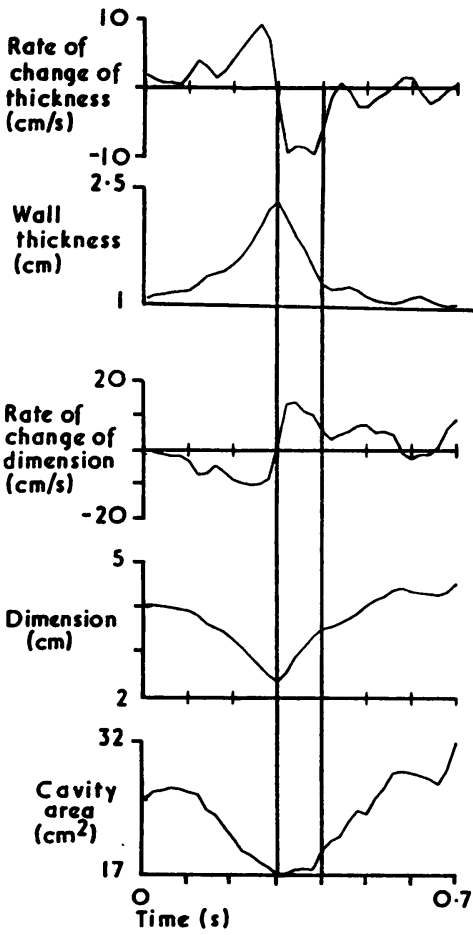

Fig. 3 Measurements of cavity area, transverse dimension, and wall thickness from a patient with ischaemic heart disease. Layout as in Fig. 1. During isovolumic relaxation, there is significant increase in dimension along with a reduction in wall thickness. 
those occurring in normal subjects (Table). For regions showing abnormal isovolumic movement, however, significant differences were present, both from normal subjects, and from normal regions of the same ventricle (Fig. 4). There was a considerable reduction of $0.8 \pm 0.1 \mathrm{~cm}$ in wall thickness before mitral valve opening $(P<0.001)$, while peak thinning rate was always recorded before mitral valve opening $(P<0.01)$, though its value was normal. As in the normal group, peak wall thickness coincided with minimum cavity area, but peak thinning rate occurred within $60 \pm 20 \mathrm{~ms}$, significantly earlier than normal $(P<0.01)$, and the rapid thinning period lasted only $120 \pm 20 \mathrm{~ms}(P<0.001)$. In these regions, the timing of the end of rapid thinning was particularly clear, since in 8 out of 10 patients, a short period of increasing wall thickness occurred at this time, approximately synchronous with mitral valve opening. This reversal was not apparent in normal regions of the same ventricle.

In 5 patients, abnormal inward movement occurred during isovolumic relaxation (Fig. 5). This was associated with $0.5 \pm 0.2 \mathrm{~cm}$ increase in wall thickness after the time of minimum cavity area, the reverse of the normal pattern. Maximum wall thickness thus followed minimum cavity area by $80 \pm 20 \mathrm{~ms}$, instead of being synchronous with it $(P<0.01)$. The duration of the rapid thinning period, and the time from maximum wall thickness to maximum thinning rate, however, were normal. In all these cases, normal peak thickening rates

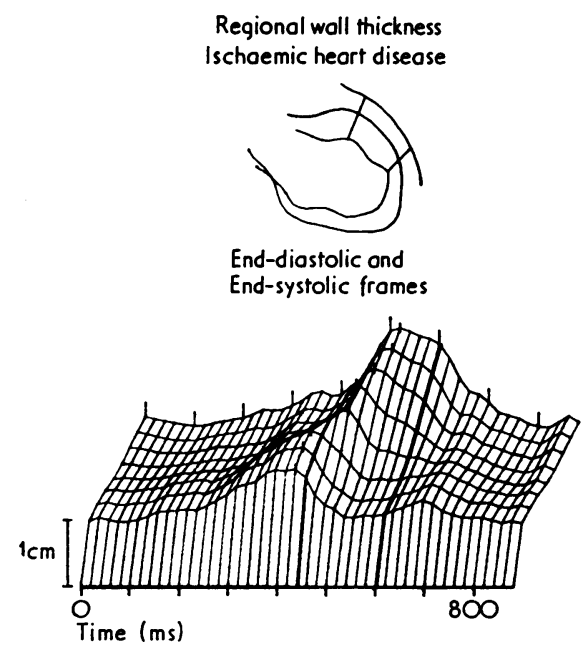

Fig. 4 Multiple plots of wall thickness against time from a patient with ischaemic heart disease. Layout as in Fig. 2. Towards the apex, there is abnormal wall thinning before mitral valve opening, but the pattern becomes normal higher up the wall. were recorded, but they were abnormal in timing, being after minimum cavity area, and thus falling in the period of isovolumic relaxation.

The relation between changes in wall thickness and dimension were investigated in the group as a whole. There was significant correlation between the reduction in transverse left ventricular dimension and the increase in wall thickness between end-diastole and end-systole, given by the equation:

(Reduction in dimension) $=1.07$ (increase in wall thickness) $+0.9 \mathrm{~cm}$

$r=0.72$, standard error of the estimate $0.25 \mathrm{~cm}$.

Ischoemic heort disease

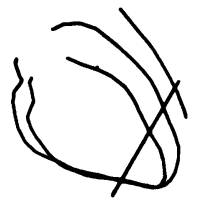

End-diastolic and End-systolic frames

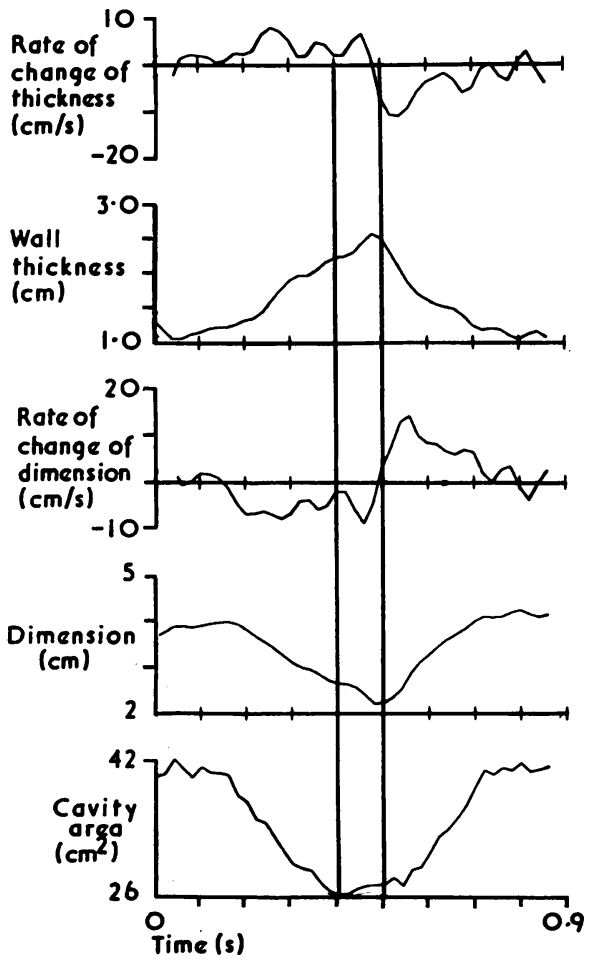

Fig. 5 Plots of cavity area, transverse dimension, and wall thickness from a patient with ischaemic heart disease. Layout as in Fig. 1. There is a reduction in dimension during isovolumic relaxation, along with an increase in wall thickness. 
Peak rates of change of dimension during systole and diastole were also correlated with the corresponding rates of change of wall thickness, the appropriate regression equations being:

$($ Peak systolic $\mathrm{dD} / \mathrm{dt})=1 \cdot 2$ (peak systolic $\mathrm{dW} / \mathrm{dt}$ ) $+2 \cdot 1 \mathrm{~cm} / \mathrm{s}$

$\mathrm{r}=0.82$, standard error of the estimate $=3.2 \mathrm{~cm} / \mathrm{s}$

(Peak diastolic $\mathrm{dD} / \mathrm{dt})=0.76$ (peak diastolic $\mathrm{dW} / \mathrm{dt}$ )

$$
+7 \cdot 1 \mathrm{~cm} / \mathrm{s}
$$

$r=0.50$, standard error of the estimate $=2.8 \mathrm{~cm} / \mathrm{s}$, where $\mathrm{D}=$ instantaneous cavity dimension, and $\mathrm{W}=$ wall thickness.

\section{Discussion}

Measurements of left ventricular wall thickness derived from angiograms differ from those obtained using ultrasound or radiographic observations of metal markers in experimental animals. Though all methods give similar estimates of wall thickness at end-diastole, at end-systole angiographic values are appreciably greater, probably because of endothelial infolding of the wall, so that sonar transducers or markers sutured to the endocardium may become separated from the cavity (Mitchell et al., 1969; Guntheroth, 1974; Rankin et al., 1976). We have made no attempt to circumvent this discrepancy. Angiography outlines the region accessible to blood throughout the cardiac cycle, and shows that this endothelial infolding is an important mechanism by which cavity size is reduced in systole. Methods based on the constancy of left ventricular mass, assumed uniformity of wall thickness, and ellipsoidal geometry at end-systole have not been used (Rackley et al., 1964; Hugenholtz et al., 1969). They would have been unsuitable for assessing abnormalities of timing, since, by definition, derived wall thickness changes must be synchronous with the corresponding changes in cavity volume. Such models are particularly unsuitable in patients with ischaemic heart disease, where the pattern of left ventricular involvement is non-uniform. Finally, on more general grounds, errors in measurement of wall thickness at endsystole result from uncertainty about endocardial rather than epicardial position, which implies corresponding uncertainty about the cavity boundary. Values of cavity size so obtained would thus be critically affected by the very uncertainty that they are being used to correct.

The present method of measuring left ventricular wall thickness has confirmed previous estimates of thickness at end-diastole and endsystole, and of peak rate of increase during systole, using comparable techniques (Eber et al., 1969; Dumesnil et al., 1974). The peak rate of change during diastole was similar to that occurring during systole, and a well-defined period of rapid thinning could be identified. In normal subjects, the timing of events in the wall was closely related to changes in cavity area (Fig. 1). Maximum wall thickness was synchronous with minimum cavity area. During isovolumic relaxation, there was a small reduction in wall thickness, but after mitral valve opening, the rate at which the wall became thinner increased, synchronous with rapid ventricular filling. The extent and timing of these changes were uniform over the region of free wall accessible to study by angiography (Fig. 2). These observations are similar to those of Rankin et al. (1976) in dogs, both in showing maximum wall thickness at end-ejection, and also a significant reduction in wall thickness during isovolumic relaxation, though Rankin found the extent of systolic wall thickening to be less, and that the minor axis tends to shorten rather than increase immediately before mitral valve opening.

A number of disturbances of left ventricular wall dynamics were present in patients with ischaemic heart disease. As has previously been found (Eber et al., 1969; Dumesnil et al., 1974), localised reduction in the amplitude of inward movement of endocardium during systole was associated with a corresponding reduction in the extent of free wall thickening, and of peak rate of increase of wall thickness during systole. More common, in the present series of patients, were disturbances of the close time relations between events in the wall and those referable to the cavity as a whole. Regions showing these disturbed time relations often behaved normally during systole, but abnormally during isovolumic relaxation, defined as the interval between minimum cavity area and mitral valve opening. These abnormal time relations of wall thickness changes closely resembled abnormalities of endocardial movement previously described (Altieri et al., 1973; Ruttley et al., 1974; Gibson et al., 1976). The latter are of two types: inward movement during isovolumic relaxation, apparently reflecting the effects of local ischaemia, and outward movement in regions with normal systolic function, representing a compensatory phenomenon. It is clear from the present study that these abnormalities, previously defined only in terms of endocardial movement, have, as their basis, localised abnormalities of wall behaviour. An abnormal increase in wall thickness after the time of minimum cavity area caused inward movement during isovolumic relaxation. Conversely, outward endocardial movement before mitral valve opening resulted from a 
premature reduction in wall thickness, with peak wall thinning rate being recorded before the start of ventricular filling. These abnormalities were confined to regions of the ventricle showing abnormal endocardial behaviour, and were not found elsewhere in the cavity where its pattern of movement was normal. Their extent was so great, particularly in regions showing outward endocardial movement during isovolumic relaxation, that they could not have been explained solely on the basis of reversal of endothelial infolding, but represented virtual completion of the diastolic reduction in wall thickness before mitral valve opening. These results thus allow previous observations to be extended (Gibson et al., 1976), and indicate that when localised ischaemia causes inward movement of endocardium during isovolumic relaxation, it does so by causing a transient increase in wall thickness rather than by any movement of epicardium. This process is associated with compensatory thinning of the wall and outward movement of endocardium in normal regions. It is these abnormalities within the wall itself that cause changes in left ventricular cavity shape previously described only in terms of their effect on endocardial position.

In normal subjects, there appeared to be a close reciprocal relation in diastole between changes in cavity size and wall thickness, with rapid ventricular filling corresponding to the period of rapid thinning. In patients showing abnormal outward movement of endocardium before mitral valve opening, however, the period of rapid wall thinning could be dissociated from that of rapid filling, since it occurred before mitral valve opening. This dissociation between events in wall and cavity suggests that rapid thinning may be as much a property of the myocardium itself as the direct result of an increase in cavity volume. Wall thinning did occur significantly more slowly, however, when it followed mitral valve opening, suggesting that in normal circumstances filling rate might exert a limiting effect on it, which was not present when it was the direct result of delayed relaxation elsewhere. Studies of changes in wall thickness in the presence of other abnormalities of ventricular filling might shed further light on this problem.

It is apparent, therefore, that measurements of wall thickness may facilitate the study of regional left ventricular function. They can be used to define localised abnormalities of cavity outline independently of any arbitrary reference point used for aligning successive cine frames, and have confirmed previous observations based on endocardial position alone. Indeed, the similarity between the two methods suggests that the theoretical possibility of significant transverse and rotational movement of the heart within the thorax is not an important one in the patients studied. In addition, abnormal wall thickness changes may themselves reflect local ischaemia, and give rise to disturbances of appreciable functional significance during isovolumic relaxation in patients with coronary artery disease. The technical resources required for these studies are modest, and their further extension seems indicated.

The computing equipment used in this study was provided by the D.H.S.S. as part of their experimental programme.

\section{References}

Altieri, P. I., Wilt, S. M., and Leighton, R. F. (1973). Left ventricular wall motion during the isovolumic relaxation period. Circulation, 48, 499-505.

Dodge, H. T., Frimer, M., and Stewart, D. K. (1974). Functional evaluation of the hypertrophied heart in man. Circulation Research, 34-35, Suppl. II, 122-127.

Dumesnil, J. G., Ritman, E. L., Frye, R. L., Gau, G. T., Rutherford, B. D., and Davis, G. D. (1974). Quantitative determination of regional left ventricular wall dynamics by Roentgen videometry. Circulation, 50, 700-708.

Eber, L. M., Greenberg, H. M., Cooke, J. M., and Gorlin, R. (1969). Dynamic changes in left ventricular free wall thickness in the human heart. Circulation, 39, 455-464.

Gibson, D. G., Prewitt, T. A., and Brown, D. J. (1976). Analysis of left ventricular wall movement during isovolumic relaxation and its relation to coronary artery disease. British Heart fournal, 38, 1010-1019.

Gould, K. L., Kennedy, J. W., Frimer, M., Pollack, G. H., and Dodge, H. T. (1976). Analysis of wall dynamics and directional components of left ventricular contraction in man. American fournal of Cardiology, 38, 322-331.

Guntheroth, W. G. (1974). Changes in left ventricular wall thickness during the cardiac cycle. fournal of Applied Physiology, 36, 208-312.

Hugenholtz, P. G., Kaplan, K., and Hull, E. (1969). Determination of left ventricular wall thickness by angiocardiography. American Heart fournal, 78, 513-522.

Mitchell, J. H., Wildenthal, K., and Mullins, C. B. (1969). Geometrical studies of the left ventricle utilizing biplane cinefluorography. Federation Proceedings, 28, 1334-1343.

Rackley, C. E., Dodge, H. T., Coble, Y. D., and Hay, R. E. (1964). Method for determining left ventricular mass in man. Circulation, 29, 666-671.

Rankin, J. S., McHale, P. A., Arentzen, C. E., Greenfield, J., Jr., and Anderson, R. W. (1976). Three dimensional dynamic geometry of the left ventricle in the conscious dog. Circulation Research, 39, 304-313.

Ruttley, M. S., Adams, D. F., Cohn, P. F., and Abrams, H. L. (1974). Shape and volume changes during 'isovolumetric relaxation' in normal and asynergic ventricles. Circulation, 50, 306-316.

Requests for reprints to Dr. D. G. Gibson, Cardiac Department, Brompton Hospital, Fulham Road, London SW3 6HP. 